\title{
A Local System for Intuitionistic Logic
}

\author{
Alwen Tiu \\ Australian National University and National ICT Australia
}

\begin{abstract}
This paper presents systems for first-order intuitionistic logic and several of its extensions in which all the propositional rules are local, in the sense that, in applying the rules of the system, one needs only a fixed amount of information about the logical expressions involved. The main source of non-locality is the contraction rules. We show that the contraction rules can be restricted to the atomic ones, provided we employ deep-inference, i.e., to allow rules to apply anywhere inside logical expressions. We further show that the use of deep inference allows for modular extensions of intuitionistic logic to Dummett's intermediate logic LC, Gödel logic and classical logic. We present the systems in the calculus of structures, a proof theoretic formalism which supports deep-inference. Cut elimination for these systems are proved indirectly by simulating the cut-free sequent systems, or the hypersequent systems in the cases of Dummett's LC and Gödel logic, in the cut free systems in the calculus of structures.
\end{abstract}

Keywords: proof theory, intuitionistic logic, intermediate logics, deep inference, calculus of structures, locality.

\section{Introduction}

This paper presents systems for intuitionistic logic and its extensions, which are properly included in classical logic, in which all the propositional rules are local, in the sense of [4]. That is, in applying the rules of the system, one needs only a fixed amount of information about the logical expressions involved. For example, the usual contraction rule in sequent calculus, i.e.,

$$
c L \frac{B, B, \Gamma \vdash C}{B, \Gamma \vdash C}
$$

is non-local, since in order to apply the rule one has to check that two formulae are syntactically equal, and since $B$ can be arbitrary formula, the "cost" of this checking varies with the size of $B$. Other examples include the (non-atomic) identity and cut rules, and the promotion rule in linear logic [11]. In [7], it is shown that it is possible to give a system for classical logic in which all the rules are local. This means in particular that the contraction, weakening, the cut and the identity rules are restricted to atomic forms. As it is shown in [5], this is difficult to achieve without some form of deep inference, i.e., to allow rules to apply anywhere inside logical expressions. The classical system in [7], called 
SKS, is presented in the calculus of structures [13], a formalism which allows deep inference in a way which preserves interesting proof theoretical notions and properties. We shall use the same formalism to present the intuitionistic systems to follow.

Deep inference and locality have been shown to allow for finer analyses on proofs, in particular, proofs in the deep-inference presentation of classical logic, i.e., the system SKS, have been shown to admit non-trivial categorical [18] and geometric interpretations [14]. Classical logic is among a number of logical systems that have been presented in the calculus of structures, e.g., noncommutative extension of linear logic [13], linear logic [21] and modal logics [20]. In these systems, the above notion of locality has been consistently exhibited. However, the logical systems in the calculus of structures studied so far have been those which are symmetric, in the sense that they have involutive negations and can be presented in one-sided sequent systems. The work presented in this paper is an attempt to find "good" presentations of asymmetric (two-sided) sequent systems in the calculus of structures, where locality is one important criteria. This will hopefully lead to further categorical or geometric models for two-sided sequent proofs for intuitionistic and intermediate logics. Another advantage of adopting deep inference is that it allows for modular presentations of several extensions of intuitionistic logic, e.g., intermediate logics and classical logic: different logical systems can be obtained by adding rules which are derived straightforwardly from the axiomatic definitions of the extended systems. Our work can hopefully serve as a basis to give a uniform presentation for various intermediate logics.

We adopt the presentation of intuitionistic logic in the calculus of structures using positive and negative contexts, due to Kai Bruennler [6] and Phillipe de Groote $^{1}$. Negative context corresponds to the left-hand side of a sequent and positive context corresponds to the right-hand side. In this presentation, rules are divided into negative rules, which apply under negative context, naturally, and positive rules which apply under positive context. Note that however since applying a rule would require checking for negative/positive context, the rules formalized this way are no longer local in the sense of [4]. But we can still achieve a weaker form of locality, that is, all rules that duplicate structures can be restricted to atomic forms. This system is then refined to a fully local one by exploiting the fact that all rule schemes in the system preserve polarities (see Section 6).

In Brünnler's intuitionistic system [6], it seems difficult, if not impossible, to reduce contraction to its atomic form. This is partly due to the fact that the contraction rule in this system (as it is the case with most sequent systems for intuitionistic logic) is asymmetric, i.e., contraction is allowed on the left (or negative context) but not on the right (positive context), while reducing contraction to its atomic form seems to require a symmetric contraction. The solution proposed here for reducing contraction to atomic is inspired by the multiple-conclusion intuitionistic system in sequent calculus $[9,23]$. In this

${ }^{1}$ The author thanks Lutz Strassburger for pointing out the contribution of de Groote. 
system, contraction and weakening are allowed on both sides of the sequent. The asymmetry of intuitionistic logic is captured by the implication rule:

$$
\supset R \frac{\Gamma, A \vdash B}{\Gamma \vdash A \supset B, \Delta}
$$

One can see for instance that the classical theorem of excluded middle, i.e., $A \vee(A \supset \perp)$, is not provable. In the calculus of structures, this is reflected by the absence of certain "logical rules" under disjunctive context (see Section 2).

There exist numerous systems for intuitionistic and intermediate logics in the literature. These systems can be roughly divided into two categories: systems which are designed with decidability and proof search in mind, e.g., contractionfree sequent systems $[16,10]$, and those which are mainly concerned with generality of the formalisms, such as labelled deduction systems [3], hypersequents [1] and display calculi [12]. Our work is more in the latter category. In terms of expressivity, the calculus of structures is certainly at least as expressive as the non-standard sequent systems (display, hypersequents, and labelled systems), as one can simulate these systems inside cut-free systems in the calculus of structures. A common feature to these extended sequent systems is that they all employ some sort of structural extensions to sequents in order to capture various extensions of intuitionistic logic. In contrast, in the calculus of structures, there is no additional structural elements added to the proof system: one simply introduces more rules to get the extended logics. Moreover, these extended rules are derived straightforwardly from their axiomatic formulations (i.e., in Hilbert's systems). However, one of the drawbacks of the formulation of deep inference systems in our work is that we currently have no "internal" proof of cut-elimination. Our cut-elimination proof is indirect via translations to other systems, notably, sequent and hypersequent systems. Methodology for proof search in deep inference systems is not yet fully developed, although there is some work in this direction [17].

The rest of the paper is organized as follows. In Section 2, we present an intuitionistic system with the general (non-local) contraction rules, called SISgq. This is then followed by the soundness and completeness proof of SISgq with respect to a multiple-conclusion sequent system for intuitionistic logic and the cut elimination proof in Section 3. Section 4 shows how to extend SISg to cover Dummett's LC, Gödel logic and classical logic. Cut elimination for LC and Gödel logic are proved indirectly by simulating the corresponding hypersequent systems for these logics $[1,2]$. In Section 5, the system SISg and its extensions are refined to systems in which the contraction rules are restricted to their atomic forms, but with additional medial rules. In Section 6 we show how to remove the context dependency in the propositional rules in all of the above logical systems, resulting in purely local systems for the propositional fragments, by introducing polarities into logical expressions. Section 7 discusses future work. Detailed proofs of the lemmas and the theorems in this paper can be found in an extended version of the paper. 


\section{An Intuitionistic System in the Calculus of Structures}

Inference rules in the calculus of structures can be seen as rewrite rules on formulae, i.e., the rules are of the form

$$
\rho \frac{F\{B\}}{F\{C\}}
$$

where $\rho$ is the name of the rule, $F\{\}$ is a formula-context and $B$ and $C$ are formulae. Basically, any sound implication $B \supset C$ can be turned into a rule. The question is of course whether doing so would result in a good proof theory. The design philosophy of the calculus of structures has been centered around the concept of interaction and symmetry in inference rules. Just as the left and right rules in sequent calculus and introduction and elimination rules in natural deduction, a rule in the calculus of structures always has its dual, called its co-rule, which is obtained from the rule by taking the contrapositive of the implication defining the rule. The concept of interaction replaces the notion of identity and cut in sequent calculus. In classical logic [4], the interaction rules are (using the standard notation for classical formulae)

$$
\text { i } \downarrow \frac{S\{\top\}}{S\{A \vee \neg A\}} \quad \text { i } \frac{S\{A \wedge \neg A\}}{S\{\perp\}}
$$

In intuitionistic logic, we shall have a slightly different notation for the interaction rules, but the idea is essentially the same: the $i \downarrow$-rule creates a dual pair of formulas (reading the rule top-down) while the $\mathrm{i} \uparrow$ rule destructs them.

In formulating the rules in the calculus of structures, one encounters certain rules which correspond to some logical equivalences in the logic being formalized. Some of the trivial equivalences, e.g., commutativity and associativity of conjunction, are more appropriately represented as equations rather than rules. We thus consider formulae modulo these equivalences. In the terms of the calculus of structures, these equivalent classes of formulae are referred to as structures. We shall be concerned with the following language of structures

$$
S:=p(t)|\mathrm{t}| \mathrm{f}|\langle S ; S\rangle|[S, S]|(S, S)| \forall x R \mid \exists x R
$$

where $p$ is a predicate symbol, $t$ is a term and the rest correspond to true, false, implication, disjunction, conjunction, universal and existential quantifications. For simplicity of presentation, we consider only unary predicates, but generalization to predicates of arbitrary arities is straightforward.

Note that we opt to use the above bracketing notations instead of the more traditional ones of connectives to simplify the presentation of the inference rules and derivations. Structures are ranged over by $R, T, U, V, W$ and atomic structures are ranged over by $a, b, c, d$. A structure context, or context for short, is a structure with a hole, denoted by $S\{\}$. Given a structure $R$ and a context $S\{\}$, we write $S\{R\}$ to denote the structure that results from replacing the hole \{\} in $S\{\}$ with $R$. In presenting a structure $R$ in a context $S\{\}$, we often omit 
Units:

$$
\begin{gathered}
{[\mathrm{t}, \mathrm{t}]=\mathrm{t} \quad(\mathrm{f}, \mathrm{f})=\mathrm{f} \quad\langle\mathrm{f} ; \mathrm{t}\rangle=\mathrm{t} \quad\langle\mathrm{f} ; \mathrm{f}\rangle=\mathrm{t}} \\
{[\mathrm{f}, R]=R \quad(\mathrm{t}, R)=R \quad\langle\mathrm{t} ; R\rangle=R} \\
{[R,[T, U]]=[[R, T], U] \quad(R,(T, U))=((R, T), U)} \\
{[R, T]=[T, R] \quad(R, T)=(T, R)} \\
\langle(R, T) ; U\rangle=\langle R ;\langle T ; U\rangle\rangle \\
\forall x . R=\exists x . R=R, \text { if } x \text { is not free in } R .
\end{gathered}
$$

Associativity:

Commutativity:

Currying:

Quantifiers:

Congruence:

$$
\forall x \cdot R=\forall y \cdot R[y / x], \exists x \cdot R=\exists y \cdot R[y / x], y \text { is not free in } \forall x . R \text {. }
$$

$$
S\{R\}=S\{T\} \text {, if } R=T \text {. }
$$

Fig. 1. Syntactic equality of structures

$$
\begin{gathered}
i \downarrow \frac{S^{+}\{\mathrm{t}\}}{S^{+}\langle R ; R\rangle} \quad \operatorname{cr} \downarrow \frac{S^{+}[R, R]}{S^{+}\{R\}} \quad \operatorname{cl} \downarrow \frac{S^{-}(R, R)}{S^{-}\{R\}} \quad \operatorname{wr} \downarrow \frac{S^{+}\{\mathrm{f}\}}{S^{+}\{R\}} \quad \operatorname{wl} \downarrow \frac{S^{-}\{\mathrm{t}\}}{S^{-}\{R\}} \\
\mathrm{s} \downarrow \frac{S^{+}([R, T], U)}{S^{+}[R,(T, U)]} \quad \operatorname{sc} \downarrow \frac{S^{+}(\langle R ; T\rangle, U)}{S^{+}\langle R ;(T, U)\rangle} \quad \operatorname{sd} \downarrow \frac{S^{+}(\langle R ; T\rangle,\langle U ; V\rangle)}{S^{+}\langle[R, U] ;[T, V]\rangle} \\
\operatorname{sid} \downarrow \frac{S^{+}[\langle R ; T\rangle, U]}{S^{+}\langle R ;[T, U]\rangle} \quad \operatorname{sic} \downarrow \frac{S^{+}(R,\langle T ; U\rangle)}{S^{+}\langle\langle R ; T\rangle ; U\rangle} \quad \operatorname{sac} \downarrow \frac{S^{+}(\forall x R, \forall x T)}{S^{+}\{\forall x(R, T)\}} \\
\operatorname{sa} \downarrow \frac{S^{+}\{\forall x\langle R ; T\rangle\}}{S^{+}\langle R ; \forall x T\rangle} \quad \operatorname{se} \downarrow \frac{S^{+}\{\forall x\langle R ; T\rangle\}}{S^{+}\langle\exists x R ; T\rangle} \quad \operatorname{nr} \downarrow \frac{S^{+}\{R[t / x]\}}{S^{+}\{\exists x R\}} \quad \mathrm{nl} \downarrow \frac{S^{-}\{R[t / x]\}}{S^{-}\{\forall x R\}}
\end{gathered}
$$

Fig. 2. System ISgq: an intuitionistic system in the calculus of structures. The rules sa $\downarrow$ and se $\downarrow$ have the provisos that $x$ is not free in $R$ and $T$, respectively.

the curly braces surrounding the $R$, if $R$ is composed with a binary relation, e.g., we shall write $S[U, V]$ instead of $S\{[U, V]\}$. Structures are considered modulo the syntactic equivalence given in Figure 1 . Note that we assume the domain of the quantification is non-empty. This is reflected in the equations concerning quantifiers.

We distinguish between positive contexts and negative contexts. Positive and negative contexts are defined inductively as follows.

1. \{\} is a positive context,

2. if $S\{\}$ is a positive context then $(S\{\}, R),(R, S\{\}),[S\{\}, R],[R, S\{\}]$, $\forall x\{\}, \exists x\{\}$ and $\langle R ; S\{\}\rangle$ are positive contexts, otherwise they are negative contexts,

3. if $S\{\}$ is a positive context then $\langle S\{\} ; R\rangle$ is a negative context, otherwise it is a positive context.

Given a positive context $S\{\}$, we often write it as $S^{+}\{\}$to emphasize that it is a positive context. Similarly we write $S^{-}\{\}$to emphasize that $S\{\}$ is a negative context.

The inference rules for the general system (non-local) for intuitionistic logic is given in Figure 2. We refer to this system as ISgq. As we have noted previously, 


$$
\begin{aligned}
& \mathrm{i} \uparrow \frac{S^{-}\langle R ; R\rangle}{S^{-}\{\mathrm{t}\}} \quad \operatorname{cr} \uparrow \frac{S^{-}\{R\}}{S^{-}[R, R]} \quad \mathrm{cl} \uparrow \frac{S^{+}\{R\}}{S^{+}(R, R)} \quad \mathrm{wr} \uparrow \frac{S^{-}\{R\}}{S^{-}\{f\}} \quad \mathrm{wl} \uparrow \frac{S^{+}\{R\}}{S^{+}\{\mathrm{t}\}} \\
& \mathrm{s} \uparrow \frac{S^{-}[R,(T, U)]}{S^{-}([R, T], U)} \quad \mathrm{sc} \uparrow \frac{S^{-}\langle R ;(T, U)\rangle}{S^{-}(\langle R ; T\rangle, U)} \quad \mathrm{sd} \uparrow \frac{S^{-}\langle[R, U] ;[T, V]\rangle}{S^{-}(\langle R ; T\rangle,\langle U ; V\rangle)} \\
& \operatorname{sid} \uparrow \frac{S^{-}\langle R ;[T, U]\rangle}{S^{-}[\langle R ; T\rangle, U]} \quad \operatorname{sic} \uparrow \frac{S^{-}\langle\langle R ; T\rangle ; U\rangle}{S^{-}(R,\langle T ; U\rangle)} \quad \operatorname{sac} \uparrow \frac{S^{-}\{\forall x(R, T)\}}{S^{-}(\forall x R, \forall x T)} \\
& \operatorname{sa} \uparrow \frac{S^{-}\langle R ; \forall x T\rangle}{S^{-}\{\forall x\langle R ; T\rangle\}} \quad \text { se } \uparrow \frac{S^{-}\langle\exists x R ; T\rangle}{S^{-}\{\forall x\langle R ; T\rangle\}} \quad \operatorname{nr} \uparrow \frac{S^{-}\{\exists x R\}}{S^{-}\{R[t / x]\}} \quad \mathrm{nl} \uparrow \frac{S^{+}\{\forall x R\}}{S^{+}\{R[t / x]\}}
\end{aligned}
$$

Fig. 3. System cISgq: the dual of ISgq

each rule in the calculus of structures has its co-rule. In the case of ISgq, the corule of a rule $\rho$ is obtained from $\rho$ by exchanging the premise with the conclusion and reversing the condition on the context of the rule (i.e., positive to negative and vice versa). The name of a rule is usually suffixed with an up or a down arrow, and its co-rule has the same name but with the arrow reversed. We use the term up-rules to denote rules with up-arrow in their names and down-rules if their names contain down-arrow. The rule $i \downarrow$ corresponds to the identity rule in sequent calculus. Its co-rule, i $\uparrow$ (see Figure 3), corresponds to cut. Together they are referred to as the interaction rules. The rules $\mathrm{cl} \downarrow$ and $\mathrm{cr} \downarrow$ are the contraction left and right rules, and $w l \downarrow$ and $w r \downarrow$ are the weakening left and right rules. The rules prefixed with the letter s are the switch rules, using the terminology of [13]. The notation $[t / x]$ in the $\mathrm{nr} \downarrow$ and $\mathrm{nI} \downarrow$ rules denotes capture-avoiding substitutions.

Notice that if we take the dual of the rules of ISgq, we obtain another, "dual" system of intuitionistic logic. This system, called clSgq, is shown in Figure 3. Each of the systems ISgq and clSgq is incomplete in its own, since either cut or identity is missing. The fully symmetric system for intuitionistic logic is thus obtained by combining the two, and is referred to as SISgq. SISgq naturally corresponds to first-order $L J$ and either one of ISgq or cISgq corresponds to the cut-free fragment of first-order $L J$. Note that either system can be chosen to represent the cut-free $L J$; it is just a matter of convention that we fix our choice to ISgq. We refer to the propositional fragment of SISgq (ISgq) as SISg (respectively, ISg).

Definition 1. A derivation $\Delta$ in a system in the calculus of structures is a finite chain of instances of inference rules in the system. A derivation can consist of just one structure. The topmost structure in a derivation is called the premise of the derivation, and the structure at the bottom is called its conclusion. A proof $\Pi$ in the calculus of structures is a derivation whose premise is t. A rule $\rho$ is derivable in a system $\mathscr{S}$ if $\rho \notin \mathscr{S}$ and for every instance of $\rho \frac{T}{R}$ there is a 
derivation with premise $R$ and conclusion $T$ in $\mathscr{S}$. Two systems are equivalent if they have the same set of provable structures.

\section{Soundness, Completeness and Cut Elimination}

We shall now prove that the system SISgq is sound and complete with respect to intuitionistic logic and that it has cut-elimination. The notion of cut-elimination in the calculus of structures is more general than that of sequent calculus, that is, not only the cut rule (the $i \uparrow$ ) is admissible, but the entire up-rules are also admissible. We prove the soundness and completeness of SISgq with respect to a multiple-conclusion sequent system for intuitionistic logic [9]. We refer to this system as $L J m$. Its rules are those of Gentzen's $L K$, except for the right introduction rules for universal quantifier and implication:

$$
\supset R \frac{\Gamma, A \vdash B}{\Gamma \vdash A \supset B, \Psi} \quad \forall R \frac{\Gamma \vdash A[y / x]}{\Gamma \vdash \forall x A, \Psi}
$$

where $y$ in the $\forall R$ rule is not free in the lower sequent. Cut-elimination for SISgq is obtained indirectly via the cut-elimination theorem in sequent calculus, by observing that all the rules in $L J m$, except the cut, are derivable in ISgq, i.e., the fragment of SISgq without the up-rules.

The formulae of $\mathrm{LJm}$ are given by the following grammar:

$$
F::=p(t)|\top| \perp|F \supset F| F \vee F|F \wedge F| \forall x F \mid \exists x F .
$$

As in structures, $p$ here denotes a unary predicate, and the rest of the constants correspond to true, false, implication, disjunction, conjunction, universal and existential quantifiers.

Definition 2. The functions.$_{S}$ and.$_{-}$given below transform formulae in LJm into structures and vice $\overrightarrow{\text { versa: }}$ :

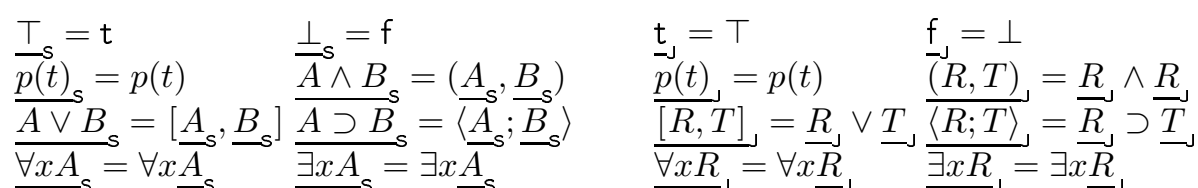

The function.$_{s}$ is generalized to sequents as follows:

$$
\underline{A_{1}, \ldots, A_{n} \vdash B}=\forall x_{1} \ldots \forall x_{n}\left\langle\left(\underline{A}_{1_{s}}, \ldots, \underline{A_{n}}\right) ; \underline{B} \underline{s}_{s}\right\rangle
$$

where $x_{1}, \ldots, x_{n}$ are the eigenvariables of the sequent, and empty conjunction is interpreted as the constant $\mathrm{t}$.

The key to proving soundness is to show that each instance of a rule in SISgq corresponds to an implication in $L J m$ and that equivalent structures map to logically equivalent formulas. For instance, the soundness of the sc $\downarrow$ rule is demonstrated by the left derivation in Figure 4. 


$$
\begin{aligned}
& i d \frac{\overline{R \vdash R} \quad i d \overline{T \vdash T}}{R \supset T, R \vdash T} \quad i d \overline{U \vdash U} \\
& \wedge R \frac{R(R \supset T, U, R \vdash T \wedge U}{(R \supset T) \wedge U, R \vdash T \wedge U} \\
& \wedge L \frac{(R \supset T)}{(R \supset T) \wedge U \vdash R \supset(T \wedge U)}
\end{aligned}
$$

$$
\begin{aligned}
& \mathrm{sc} \downarrow \frac{\left(\left\langle\Gamma_{1} ;\left[A, \Psi_{2}\right]\right\rangle,\left\langle\left(B, \Gamma_{2}\right) ; \Psi_{1}\right\rangle\right)}{\left\langle\Gamma_{1} ;\left(\left[A, \Psi_{2}\right],\left\langle\left(B, \Gamma_{2}\right) ; \Psi_{1}\right\rangle\right)\right\rangle} \\
& \mathrm{sc} \downarrow \frac{\left\langle\Gamma_{1} ;\left(\left\langle\Gamma_{2} ;\left\langle B ; \Psi_{1}\right\rangle\right\rangle,\left[A, \Psi_{2}\right]\right)\right\rangle}{\left\langle\Gamma_{1} ;\left\langle\Gamma_{2} ;\left(\left\langle B ; \Psi_{1}\right\rangle,\left[A, \Psi_{2}\right]\right)\right\rangle\right\rangle} \\
& =\frac{\left\langle\left(\Gamma_{1}, \Gamma_{2}\right) ;\left(\left[A, \Psi_{2}\right],\left\langle B ; \Psi_{1}\right\rangle\right)\right\rangle}{\left\langle\left(\Gamma_{1}, \Gamma_{2}\right) ;\left\langle\left(A,\left\langle B ; \Psi_{1}\right\rangle\right), \Psi_{2}\right]\right\rangle} \\
& \mathbf{s} \downarrow \frac{\left\langle\left(\Gamma_{1}, \Gamma_{2}\right) ;\left[\left(A,\left\langle B ; \Psi_{1}\right\rangle\right), \Psi_{2}\right]\right\rangle}{\left.\left.\left\langle\left(\Gamma_{1}, \Gamma_{2}\right) ;\langle A ; B\rangle ; \Psi_{1}\right\rangle, \Psi_{2}\right]\right\rangle} \\
& \operatorname{sic} \downarrow \frac{\left\langle\left(\Gamma_{1}, \Gamma_{2}\right) ;\left[\left\langle\langle A ; B\rangle ; \Psi_{1}\right\rangle, \Psi_{2}\right]\right\rangle}{\left\langle\left(\Gamma_{1}, \Gamma_{1}\right) ;(A, j, ;\right.} \\
& \operatorname{sid} \downarrow \frac{\downarrow}{\left\langle\left(\Gamma_{1}, \Gamma_{2}\right) ;\left\langle\langle A ; B\rangle ;\left[\Psi_{1}, \Psi_{2}\right]\right\rangle\right\rangle}
\end{aligned}
$$

Fig. 4. A correspondence between ISgq and $L J m$

Theorem 3. For every structure $R, R$ is provable in SISgq if and only if $R$ is provable in LJm.

To prove completeness, and cut-elimination, we show that ISgq can simulate all the sequent rules of $\mathrm{LJm}$. For instance, we show in the right derivation in Figure 4 a simulation of the left introduction rule for implication:

$$
\supset L \frac{\Gamma_{1} \vdash A, \Psi_{1} \quad B, \Gamma_{2} \vdash \Psi_{2}}{\Gamma_{1}, \Gamma_{2}, A \supset B \vdash \Psi_{1}, \Psi_{2}}
$$

Notice that the branching in the rule is mapped to the conjunctive structural relation in ISgq.

Theorem 4. For every structure $R, R$ is provable in SISgq if and only if it is provable in ISgq.

\section{Intermediate and Classical Logics}

We now consider three extensions of intuitionistic logic: Dummett's $L C$ [8], Gödel logic [22] and classical logic. Dummett's $L C$ is obtained by adding the following axiom $A \supset B \vee B \supset A$ to the propositional fragment of intuitionistic logic. Gödel logic is obtained by adding to $L C$ the axiom $\forall x(A \vee B) \supset \forall x A \vee B$, where $x$ is not free in $B$. Classical logic is obtained, obviously, by dropping the restriction on the contexts in the introduction rules for implication and universal quantifiers. We discuss each of these extension in the following.

\subsection{Dummett's $L C$}

Dummett's $L C$ can be formalized in the calculus of structures by adding the following rules to SISg (i.e., the propositional fragment of SISgq).

$$
\operatorname{com} \downarrow \frac{S^{+}(\langle R ; T\rangle,\langle U ; V\rangle)}{S^{+}[\langle R ; V\rangle,\langle U ; T\rangle]} \quad \operatorname{com} \uparrow \frac{S^{-}[\langle R ; V\rangle,\langle U ; T\rangle]}{S^{-}(\langle R ; T\rangle,\langle U ; V\rangle)}
$$


These rules are called the communication rules, and are inspired by the corresponding rules in the hypersequent formulation of $L C[1,2]$. With the com $\downarrow$ rule, we can derive the axiom $A \supset B \vee B \supset A$ as follows:

$$
\begin{gathered}
=\frac{\mathrm{t}}{(\mathrm{t}, \mathrm{t})} \\
\mathrm{i} \downarrow \frac{(\mathrm{t},\langle B ; B\rangle)}{(\langle A ; A\rangle,\langle B ; B\rangle)} \\
\operatorname{com} \downarrow \frac{(\langle A ; B\rangle,\langle B ; A\rangle]}{[\langle A ;}
\end{gathered}
$$

We refer to the system SISg extended with both rules as SCSg. We call the down fragment of SCSg, i.e., ISg plus the com $\downarrow$ rule, the system CSg. As we will see, it is enough to consider CSg since it is equivalent to SCSg.

Both com $\downarrow$ and com $\uparrow$ rules correspond to the formula

$$
(R \supset T) \wedge(U \supset V) \supset(R \supset V) \vee(U \supset T) .
$$

This formula can be easily shown to be provable from the following three formulas:

1. $(T \supset V) \vee(V \supset T)$,

2. $(R \supset T) \wedge(T \supset V) \supset(R \supset V)$,

3. $(U \supset V) \wedge(V \supset T) \supset(U \supset T)$.

The first formula is an axiom of $L C$, the second and the third are intuitionistic theorems. Therefore the com $\downarrow$ and com $\uparrow$ rules are sound with respect to $L C$. The completeness proof of CSg (and SCSg) is more involved; it uses a translation from a hypersequent system for $L C$ to $\mathrm{CSg}$. We state the result here and refer the interested reader to the extended version of the paper for the detailed proofs.

Theorem 5. For every structure $R, R$ is provable in $\mathrm{CSg}$ if and only if $R$ is provable in $\mathrm{LC}$.

\subsection{Gödel Logic}

Gödel logic is obtained by adding com $\downarrow$, com $\uparrow$ and the following rules to SISgq:

$$
\mathrm{g} \downarrow \frac{S^{+}\{\forall x[R, T]\}}{S^{+}[\forall x R, T]} \quad \mathrm{g} \uparrow \frac{S^{-}[\forall x R, T]}{S^{-}\{\forall x[R, T]\}}
$$

We refer to the formulation of this logic as SGSg. The down fragment, i.e., CSg plus the $g \downarrow$ rule, is referred to as GSg.

The rules $\mathrm{g} \downarrow$ and $\mathrm{g} \uparrow$ are obviously sound since they correspond directly to the axiom $\forall x(R \vee T) \supset \forall x R \vee T$. To prove completeness and cut-elimination, we encode a hypersequent system for Gödel logic, i.e., the system HIF [2] (also known as first-order intuitionistic fuzzy logic) in GSg. The details of the encoding can be found in the appendix.

Theorem 6. For every structure $R, R$ is provable in GSg if and only if $R$ is provable in $H I F$. 


\subsection{Classical Logic}

Classical logic is obtained by adding $\mathrm{g} \downarrow$, $\mathrm{g} \uparrow$ and the following rules

$$
\text { ci } \downarrow \frac{S^{+}\langle R ;[T, U]\rangle}{S^{+}[\langle R ; T\rangle, U]} \quad \operatorname{ci} \uparrow \frac{S^{-}[\langle R ; T\rangle, U]}{S^{-}\langle R ;[T, U]\rangle}
$$

to SISgq. These rules allow one to simulate the right-introduction rules for implication and universal quantifier in $L K$ :

$$
\supset R \frac{\Gamma, A \vdash B, \Psi}{\Gamma \vdash A \supset B, \Psi} \quad \forall R \frac{\Gamma \vdash A[y / x], \Psi}{\Gamma \vdash \forall x A, \Psi}
$$

More precisely, these rules are derived as follows:

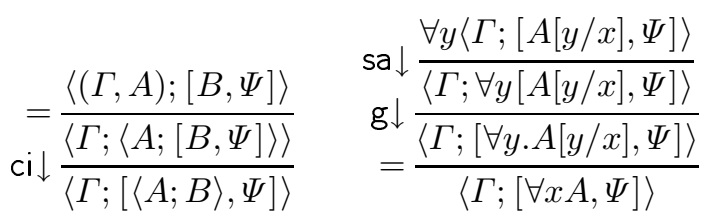

We refer to the system SISgq extended with ci $\downarrow$, ci $\uparrow, g \downarrow$ and $g \uparrow$ as $\mathrm{SKS}_{2 \mathrm{~g}}$. The down fragment, i.e., ISgq extended with ci $\downarrow$ and $g \downarrow$, is referred to as $\mathrm{KS}_{2 \mathrm{~g}}$.

Theorem 7. For every structure $R, R$ is provable in $\mathrm{KS}_{2 \mathrm{~g}}$ if and only if $\underline{R}_{\mathrm{J}}$ is provable in $L K$.

\section{Atomic Contraction}

We shall now refine the system SISgq and its extensions to systems in which the interaction rules (i.e., the $i \downarrow$ and $\mathrm{i} \uparrow$ rules), contraction and weakening are restricted to atomic propositions. The transformations required to reduce the interaction, weakening and contraction rules to their atomic forms are independent of the particular extensions to SISgq, so without loss of generality we shall work only with the system SISgq in this section. The main challenge in reducing contraction to its atomic form is in finding the right medial rules, just like those in SKS [4]. They are basically some form of distributivity among the connectives. In order to reduce contraction in negative context to atomic ones, it is crucial that we allow contraction on positive context as well. This is due to the reversal of polarity introduced by the implication connective.

The atomic versions of the interaction, contraction and weakening rules are as follows:

$$
\text { ai } \downarrow \frac{S^{+}\{\mathrm{t}\}}{S^{+}\langle a ; a\rangle} \quad \operatorname{acr} \downarrow \frac{S^{+}[a, a]}{S^{+}\{a\}} \quad \operatorname{acl} \downarrow \frac{S^{-}(a, a)}{S^{-}\{a\}} \quad \operatorname{awr} \downarrow \frac{S^{+}\{\mathrm{f}\}}{S^{+}\{a\}} \quad \text { awl } \downarrow \frac{S^{-}\{\mathrm{t}\}}{S^{-}\{a\}}
$$

and their respective duals, obtained by exchanging the premise and the conclusion, with the polarity of the context reversed. Here we denote with $a$ an atomic formula. 


$$
\begin{array}{rc}
\mathrm{ml} \frac{S^{-}([R, T],[U, V])}{S^{-}[(R, U),(T, V)]} & \mathrm{mr} \frac{S^{+}[(R, U),(T, V)]}{S^{+}([R, T],[U, V])} \\
\operatorname{mil} \downarrow \frac{S^{-}(\langle R ; U\rangle,\langle T ; V\rangle)}{S^{-}\langle[R, T] ;(U, V)\rangle} & \operatorname{mir} \downarrow \frac{S^{+}[\langle R ; U\rangle,\langle T ; V\rangle]}{S^{+}\langle(R, T) ;[U, V]\rangle} \\
\operatorname{mal} \downarrow \frac{S^{-}(\forall x R, \forall x T)}{S^{-}\{\forall x(R, T)\}} & \operatorname{mar} \downarrow \frac{S^{+}[\forall x R, \forall x T]}{S^{+}\{\forall x[R, T]\}} \\
\operatorname{mel} \downarrow \frac{S^{-}(\exists x R, \exists x T)}{S^{-}\{\exists x(R, T)\}} & \operatorname{mer} \downarrow \frac{S^{+}[\exists x R, \exists x T]}{S^{+}\{\exists x[R, T]\}}
\end{array}
$$

Fig. 5. The medial rules for reducing contraction to atomic

The medial rules for intuitionistic logic are given in Figure 5. The classical medial rule $\mathrm{m}$ of SKS [7] splits into two rules: the (right) medial rule $\mathrm{mr}$ and the (left) medial rule $\mathrm{ml}$. This is because we have contraction on both the positive and negative contexts. Notice that $\mathrm{mr}$ and $\mathrm{ml}$ are dual to each other, that is, $\mathrm{mr}$ is the up-version of $\mathrm{ml}$ and vice versa. There are extra medial rules that deal with implication and quantifiers. All those rules are derivable from the contraction and weakening rules in ISgq, and hence their soundness follows from the soundness of ISgq. By taking the duals of the medial rules in Figure 5, we obtain the co-medial rules, which by symmetry, are needed to reduce the co-contraction (i.e., the upversion of the contraction rules) to atomic. The co-medial rules are denoted by the same name but with the arrows reversed.

The general interaction rules $i \downarrow$ and the weakening rule $w r \downarrow$ can be shown reducible to their atomic versions, and the contraction rule $\mathrm{cr} \downarrow$ can be reduced to the atomic one with the medial rules. We illustrate here a step in the reduction of the contraction rule; more details can be found in the appendix. Consider for instance, contractions on an implication structure, on both the positive and negative context:

$$
\operatorname{cr} \downarrow \frac{S^{+}[\langle R ; T\rangle,\langle R ; T\rangle]}{S^{+}\langle R ; T\rangle} \quad \operatorname{cl} \downarrow \frac{S^{-}(\langle R ; T\rangle,\langle R ; T\rangle)}{S^{-}\langle R ; T\rangle}
$$

These instances of contractions can be replaced by the following derivations:

$$
\begin{array}{rr}
\operatorname{mir} \downarrow & \frac{S^{+}[\langle R ; T\rangle,\langle R ; T\rangle]}{S^{+}\langle(R, R) ;[T, T]\rangle} \\
\operatorname{cr} \downarrow \frac{\operatorname{mil}}{} & \operatorname{cl} \frac{S^{-}[\langle R ; T\rangle,\langle R ; T\rangle]}{S^{-}\langle[R, R] ;(T, T)\rangle} \\
\quad \operatorname{cl} \downarrow \frac{S^{+}\langle(R, R) ; T\rangle}{S^{+}\langle R ; T\rangle} & \operatorname{cr} \downarrow \frac{S^{-}\langle[R, R] ; T\rangle}{S^{-}\langle R ; T\rangle}
\end{array}
$$

Notice that in the above derivations, contractions are applied to a subformula of the original formula. Repeating this process, we eventually end up with contractions on atomic formulas only.

Definition 8. System ISaq is obtained from ISgq by replacing the interaction rule $\mathrm{i} \downarrow$ with ai $\downarrow$, the weakening rules $\mathrm{wr} \downarrow$ and $\mathrm{wl} \downarrow$ with awr $\downarrow$ and awl $\downarrow$, the 
Units:

$$
\begin{aligned}
& {\left[\mathrm{t}^{+}, \mathrm{t}^{+}\right]^{+}=\mathrm{t}^{+} \quad\left(\mathrm{f}^{+}, \mathrm{f}^{+}\right)^{+}=\mathrm{f}^{+} \quad\left\langle\mathrm{f}^{-} ; \mathrm{t}^{+}\right\rangle^{+}=\mathrm{t}^{+} \quad\left\langle\mathrm{f}^{-} ; \mathrm{f}^{+}\right\rangle^{+}=\mathrm{t}^{+}} \\
& {\left[\mathrm{f}^{+}, R^{+}\right]^{+}=R^{+} \quad\left(\mathrm{t}^{+}, R^{+}\right)^{+}=R^{+}\left\langle\mathrm{t}^{-} ; R^{+}\right\rangle^{+}=R^{+}} \\
& \text {Associativity: } \quad\left[R,[T, U]^{+}\right]^{+}=\left[[R, T]^{+}, U\right]^{+} \quad\left(R,(T, U)^{+}\right)^{+}=\left((R, T)^{+}, U\right)^{+} \\
& \text {Commutativity: } \quad[R, T]^{+}=[T, R]^{+} \quad(R, T)^{+}=(T, R)^{+} \\
& \left\langle(R, T)^{-} ; U\right\rangle^{+}=\left\langle R ;\langle T ; U\rangle^{+}\right\rangle^{+} \\
& \bar{R}=\bar{T} \text {, if } R=T \text {. } \\
& S\{R\}=S\{T\} \text {, if } R=T \text {. }
\end{aligned}
$$$$
\text { Currying: }
$$$$
\text { Orthogonality: }
$$$$
\text { Congruence: }
$$

Fig. 6. Syntactic equality for polarized structures

contraction rules $\mathrm{cr} \downarrow$ and $\mathrm{cl} \downarrow$ with $\mathrm{acr} \downarrow$, acl $\downarrow$ and the medial rules in Figure 5. System SISaq is obtained by adding to ISaq its own dual rules. The propositional fragment of SISaq and ISaq are referred to as SISa and ISa, respectively.

Theorem 9. The systems SISgq, SISaq, SISaq and ISaq are equivalent.

\section{A Local System for Propositional Intuitionistic Logic}

The rules in both SISgq and SISaq are non-local since in order to apply the rules, one has to check whether the redex is in a positive or negative context. However, if one carefully observes the rules, one notices a certain conservation of polarities in the rules. That is to say there is never the case where a structure in a positive context is moved to a negative context and vice versa. For example, in the rule sc $\downarrow$ in Figure 2, the substructures $R, T, U$ and $V$ have the same polarities in both the premise and the conclusion of the rule. That is $R$ is in negative context in both premise and conclusion, $T$ is in positive context, and so on. This observation leads to the following idea: When proving a structure, we first label each substructure with either a ' +' or a ' - ' depending on whether the substructure is in a positive or a negative context respectively. Each time a structure is modified by a rule, the premise of the rule is relabelled consistently, that is, substructures are labelled depending on which context they reside in. The polarity-preserving property of the rules guarantees that there is no need of relabelling of substructures which are not affected by the rule. For the sc $\downarrow$ rule, the labelled version would be:

$$
\mathrm{sc} \downarrow \frac{S\left(\langle R ; T\rangle^{+}, U\right)^{+}}{S\left\langle R ;(T, U)^{+}\right\rangle^{+}}
$$

This modified rule of sc $\downarrow$ is local since we need only to check for polarity of three substructures in the rule, instead of checking the entire context. We shall give a fully local system for the propositional fragment of ISaq by introducing polarities into structures. 


$$
\begin{aligned}
& \text { ai } \downarrow \frac{S\left\{\mathrm{t}^{+}\right\}}{S\left\langle a^{-} ; a^{+}\right\rangle^{+}} \quad \operatorname{acr} \downarrow \frac{S\left[a^{+}, a^{+}\right]^{+}}{S\left\{a^{+}\right\}} \quad \operatorname{acl} \downarrow \frac{S\left(a^{-}, a^{-}\right)^{-}}{S\left\{a^{-}\right\}} \quad \operatorname{awr} \downarrow \frac{S\left\{\mathrm{f}^{+}\right\}}{S\left\{a^{+}\right\}} \quad \mathrm{wl} \downarrow \frac{S\left\{\mathrm{t}^{-}\right\}}{S\left\{a^{-}\right\}} \\
& \mathrm{s} \downarrow \frac{S\left([R, T]^{+}, U\right)^{+}}{S\left[R,(T, U)^{+}\right]^{+}} \quad \mathrm{sc} \downarrow \frac{S\left(\langle R ; T\rangle^{+}, U\right)^{+}}{S\left\langle R ;(T, U)^{+}\right\rangle^{+}} \quad \mathrm{sd} \downarrow \frac{S\left(\langle R ; T\rangle^{+},\langle U ; V\rangle^{+}\right)^{+}}{S\left\langle[R, U]^{-} ;[T, V]^{+}\right\rangle^{+}} \\
& \operatorname{sid} \downarrow \frac{S\left[\langle R ; T\rangle^{+}, U\right]^{+}}{S\left\langle R ;[T, U]^{+}\right\rangle^{+}} \quad \operatorname{sic} \downarrow \frac{S\left(R,\langle T ; U\rangle^{+}\right)^{+}}{S\left\langle\langle R ; T\rangle^{+} ; U\right\rangle^{+}} \\
& \mathrm{ml} \frac{S\left([R, T]^{-},[U, V]^{-}\right)^{-}}{S\left[(R, U)^{-},(T, V)^{-}\right]^{-}} \quad \mathrm{mr} \frac{S\left[(R, U)^{+},(T, V)^{+}\right]^{+}}{S\left([R, T]^{+},[U, V]^{+}\right)^{+}} \\
& \operatorname{mil} \downarrow \frac{S\left(\langle R ; U\rangle^{-},\langle T ; V\rangle^{-}\right)^{-}}{S\left\langle[R, T]^{+} ;(U, V)^{-}\right\rangle^{-}} \quad \operatorname{mir} \downarrow \frac{S\left[\langle R ; U\rangle^{+},\langle T ; V\rangle^{+}\right]^{+}}{S\left\langle(R, T)^{-} ;[U, V]^{+}\right\rangle^{+}}
\end{aligned}
$$

Fig. 7. System ISp

Definition 10. Polarized structures are expressions generated from the following grammar:

$$
\begin{aligned}
& S::=P \mid N \\
& P::=a^{+}\left|\mathrm{t}^{+}\right| \mathrm{f}^{+}\left|(P, P)^{+}\right|[P, P]^{+} \mid\langle N ; P\rangle^{+} \\
& N::=a^{-}\left|\mathrm{t}^{-}\right| \mathrm{f}^{-}\left|(N, N)^{-}\right|[N, N]^{-} \mid\langle P ; N\rangle^{-}
\end{aligned}
$$

A positive polarized structure, or positive structure for short, is a polarized structure labelled with '+', and a negative polarized structure, or negative structure, is a polarized structure labelled with '-'. Positive structures are often denoted by $R^{+}$and negative structures by $R^{-}$. The orthogonal of a structure $R$, denoted by $\bar{R}$, is the structure obtained from $R$ by exchanging the labels '+' with '-' and vice versa. A polarized context is a polarized structure with a hole \{\} . Given a polarized context $S\{\}$ and a polarized structure $R$, the placement of $R$ in $S\{\}$, i.e., $S\{R\}$, is allowed only if doing so results in a well-formed polarized structure. Polarized structures are considered modulo the equality in Figure 6.

The propositional intuitionistic system with polarized structures is given in Figure 7. We refer to this system as ISp. Each polarized rule has a dual version which is obtained by exchanging the premise and the conclusion and exchanging the polarities. The system obtained by adding ISp to its own duals is is referred to as SISp. Both the inference rules and the structural equality are derived straightforwardly from the inference rules and structural equality of SISa, that is, by giving appropriate labels to the structures. Care has to be taken to ensure that the rules and the equality between polarized structures preserve polarity. We shall now proceed to prove formally that SISp, SISa, ISp and ISa are all equivalent in terms of provability.

The notion of derivations in SISp is the same as that in SISa. The notion of proof is slightly different. 
Definition 11. A proof of a polarized structure $R$ in $\mathscr{S}$ is a derivation in $\mathscr{S}$ with premise $\mathrm{t}^{+}$and conclusion $R$.

By this definition, it is obvious that all provable polarized structures are positive structures since all rules in SISp preserve polarities.

The key idea to proving the correspondence between SISp and the propositional fragment of SISaq is the following: the polarity of any substructure $R$ in $S\{R\}$ should determine the polarity of the context. In particular, positive structures $R$ and $S\{R\}$ are translated to some structures $T$ and $S^{\prime}\{T\}$ such that $S^{\prime}\{\}$ corresponds to $S\{\}$ and $T$ corresponds to $R$, and most importantly, $S^{\prime}\{\}$ is a positive context. In this way, rules that apply to positive substructures in SISp translate to the same rules that apply under positive context in SISa, and a simple observation on the inference rules of SISp and SISa shows that they co-incide. The same observation holds for negative structures and negative contexts. In the following theorems, we denote with $R$, where $R$ is a polarized structure, the structure obtained from $R$ by dropping all the polarity signs.

Theorem 12. For every polarized structure $R, R$ is provable in ISp if and only if $\underline{R}_{\mathrm{s}}$ is provable in ISa.

\section{Future Work}

Properties of proofs and derivations in the systems SISgq and its extensions remain to be studied. An immediate future works would be to find direct proofs (i.e., without the detour through sequent calculus or hypersequent) of cutelimination. It would also be interesting to investigate various substructural logics that arise from either restricting or extending the base system SISgq. For instance, it would be interesting to see what sort of logic we get from dropping the atomic contraction rules but keeping the medial rules. Another open problem is to come up with a fully local first-order intuitionistic system. The rules which instantiate quantifiers, i.e., $\mathrm{nr} \downarrow$ and $\mathrm{nl} \downarrow$, involve substitutions which are non-local. This can probably be made local by giving rules which effectively "implement" explicit substitutions. On the more general problem of formalizing asymmetric systems, it would be intereting to see if the methodology presented here can be generalized to formalize non-standard asymmetric systems such as Bunched Logic [19]. Some preliminary result in this direction can be found in [15]. The current work focusses mainly on the proof theoretic aspects. It would be interesting to see if the analyses on the deep inference systems, in particular the notions of locality and atomicity, will be useful for implementing proof search for these logics.

Acknowledgement. The author would like to thank Alessio Guglielmi for his useful comments and suggestions, in particular the one concerning the use of polarities. The author would also like to thank Kai Brünnler, Lutz Strassburger and the anonymous referees for their useful comments and suggestions on earlier drafts of the paper. The author is grateful for the support he received from INRIA Lorraine/LORIA during the start of this work in 2005. 


\section{References}

1. A. Avron. Hypersequents, logical consequence and intermediate logics for concurrency. Ann. Math. Artif. Intell., 4:225-248, 1991.

2. M. Baaz and A. Ciabattoni. A Schütte-Tait style cut-elimination proof for firstorder Gödel logic. In U. Egly and C. G. Fermüller, editors, TABLEAUX, volume 2381 of Lecture Notes in Computer Science, pages 24-37. Springer, 2002.

3. V. Balat and D. Galmiche. Labelled proof systems for intuitionistic provability. pages $1-32,2000$.

4. K. Brünnler. Deep Inference and Symmetry in Classical Proofs. PhD thesis, TU Dresden, September 2003.

5. K. Brünnler. Two restrictions on contraction. Logic Journal of the IGPL, 11(5):525-529, 2003.

6. K. Brünnler. Intuitionistic logic in the calculus of structures. Unpublished notes, 2004.

7. K. Brünnler and A. F. Tiu. A local system for classical logic. In R. Nieuwenhuis and A. Voronkov, editors, LPAR 2001, volume 2250 of LNCS, pages 347-361. Springer-Verlag, 2001.

8. M. Dummett. A propositional calculus with denumerable matrix. J. Symbolic Logic, 24(2):97-106, 1959.

9. M. Dummett. Element of Intuitionism. Oxford University Press, 1977.

10. R. Dyckhoff. Contraction-free sequent calculi for intuitionistic logic. Journal of Symbolic Logic, 57(3):795-807, 1992.

11. J.-Y. Girard. Linear logic. Theoretical Computer Science, 50:1-102, 1987.

12. R. Goré. Substructural logics on display. Logic Journal of the IGPL, 6(3):451-504, 1998.

13. A. Guglielmi. A system of interaction and structure. Technical Report WV-02-10, TU Dresden, 2002. Accepted by ACM Transactions on Computational Logic.

14. Y. Guiraud. The three dimensions of proofs. To appear in Annals of Pure and Applied Logic, 2006.

15. B. Horsfall. Towards BI in the calculus of structures. Accepted at ESSLLI 2006.

16. J. Hudelmaier. Bounds on cut-elimination in intuitionistic propositional logic. Archive for Mathematical Logic, 31:331-354, 1992.

17. O. Kahramanoğulları. Reducing nondeterminism in the calculus of structures. Accepted at LPAR 2006.

18. R. McKinley. Classical Categories and Deep Inference. PhD thesis, University of Bath, 2006.

19. P. O'Hearn and D. Pym. The logic of bunched implications. Bulletin of Symbolic Logic, 5(2):215-243, June 1999.

20. C. Stewart and P. Stouppa. A systematic proof theory for several modal logics. In R. Schmidt, I. Pratt-Hartmann, M. Reynolds, and H. Wansing, editors, Advances in Modal Logic, volume 5 of King's College Publications, pages 309-333, 2005.

21. L. Straßburger. Linear Logic and Noncommutativity in the Calculus of Structures. PhD thesis, Technische Universität Dresden, 2003.

22. G. Takeuti and S. Titani. Intuitionistic fuzzy logic and intuitionistic fuzzy set theory. J. Symbolic Logic, 49(3):851-866, 1984.

23. A. Troelstra and H. Schwichtenberg. Basic Proof Theory. Cambridge University Press, 1996. 